\title{
THE SIDON CONSTANT OF A FINITE ABELIAN GROUP
}

\author{
COLIN C. GRAHAM
}

Abstract. It is shown that the Helson constant of a finite abelian group, $G$, is exactly $(\text { Card } G)^{1 / 2}$.

The purpose of this note is to prove the following theorem.

THEOREM. Let $G$ be a finite abelian group of cardinality $n$. Then there exists a nonzero measure $\mu$ on $G$ such that $\|\mu\| /\|\hat{\mu}\|_{\infty}=n^{1 / 2}$.

The Sidon (or Helson) constant $\alpha(E)$ of a finite set $E$ of a locally compact abelian group is the supremum of the ratio $\|\mu\| /\|\hat{\mu}\|_{\infty}$ as $\mu$ ranges over nonzero measures concentrated on $E$. The Sidon constant for $E$ is at most $(\text { Card } E)^{1 / 2}$ (see [K, p. 34]). Thus, the theorem establishes the claim of the abstract.

This result is a qualitative improvement of previous results. For example, [LR, pp. 78-80] shows that the Sidon constant of a finite abelian group is at least (Card $G / 2 e \log \operatorname{Card} G)^{1 / 2}$. For a finite cyclic group, Shapiro-Rudin polynomials can be used to show that the Sidon constant of $G$ is at least $2^{-3 / 2}(\operatorname{Card} G)^{1 / 2}[K$, p. 35]. Neither of these results can be improved by modification of the techniques used to obtain them.

ProOF OF THEOREM. It will be sufficient to prove the theorem in case that $G$ is a finite cyclic group. Indeed, if the theorem holds for finite cyclic groups, and $G$ is a finite product of cyclic groups, then the product of the measures "that work" for the factors of $G$ has the required property.

We now exhibit the measure that has the required property in case that $G$ is a finite cyclic group of order $n$. We identify $G$ with the integers $1,2, \ldots, n$ with addition modulo $n$.

If $n$ is even, we let $\mu$ be the measure on $G$ that has mass at $j$ given by

$$
\mu(j)=\exp \left(2 \pi i j^{2} / 2 n\right), \quad \text { for } 1 \leqslant j \leqslant n .
$$

Obviously $\|\mu\|=n$. We need to show that $\|\hat{\mu}\|_{\infty}=n^{1 / 2}$. Since $\left\|(\mu * \tilde{\mu})^{\wedge}\right\|_{\infty}$ $=\|\hat{\mu}\|_{\infty}^{2}$, it will suffice to show that $\mu * \mu=n \delta$ where $\delta$ is the point mass at the identity. We calculate. The following formulae are easily established. (Recall that addition is $\bmod n$.)

Received by the editors May 20, 1977.

AMS (MOS) subject classifications (1970). Primary 42A12, 43A25; Secondary 46B10.

Key words and phrases. Sidon constant for finite abelian groups, Shapiro-Rudin polynomials, Fourier transform of a measure on a finite abelian group, Helson constant for finite abelian group. 


$$
\mu(-j)=\mu(n-j)=\mu(j) .
$$

$$
\tilde{\mu}(j)=\exp \left(-2 \pi i j^{2} / 2 n\right) \text {. }
$$

We then have, for $1<k<n$,

$$
\begin{aligned}
\mu * \tilde{\mu}(k) & =\sum_{j=1}^{n} \mu(k-j) \tilde{\mu}(j) \\
& =\sum_{j=1}^{n} \exp \left(2 \pi i\left[(k-j)^{2}-j^{2}\right] / 2 n\right) \\
& =\exp \left(2 \pi i k^{2} / 2 n\right) \sum_{j=1}^{n} \exp (2 \pi i(-k j / n)) .
\end{aligned}
$$

Of course, when $1<k<n$, the last sum is zero. Thus, $\mu * \mu=n \delta$.

For odd $n$, we use $\mu(j)=\exp \left(2 \pi i j^{2} / n\right)$. Then $\tilde{\mu}(j)=\exp \left(-2 \pi i j^{2} / n\right)$ and

$$
\mu * \tilde{\mu}(k)=\exp \left(2 \pi i k^{2} / n\right) \sum_{j=1}^{n} \exp (2 \pi i 2 k j / n) .
$$

Since, for odd $n, 2 k \equiv 0(\bmod n)$ if and only if $k \equiv 0(\bmod n)$, the last sum is zero when $1<k<n$. Thus, $\mu * \tilde{\mu}=n \delta$.

REMARK. The corresponding problem for arithmetic progressions is much more difficult. It is not known if $\lim \alpha(\{1,2, \ldots, n\}) / n^{1 / 2}=1$. See [N] for a discussion.

\section{REFERENCES}

[K] J.-P. Kahane, Séries de Fourier absolument convergentes, Springer-Verlag, Berlin and New York, 1970.

[LR] J. López and K. A. Ross, Sidon sets, Dekker, New York, 1975.

[N] D. J. Newman, An $L^{1}$-extremal problem for polynomials, Proc. Amer. Math. Soc. 16 (1965), 1287-1290.

Department of Mathematics, NorthWestern University, Evanston, Illinois 60201 